\title{
sciendo
}

\section{ASSOCIATION OF SNPS IN AKIRIN2, TTN, EDG1 AND MYBPC1 GENES WITH GROWTH AND CARCASS TRAITS IN QINCHUAN CATTLE*}

\author{
Zixuan Cheng ${ }^{1 *}$, Cong Sheng ${ }^{24}$, Yaxing $\mathrm{Li}^{1}$, Shenyuan Wang ${ }^{3}$, Kaifeng $\mathrm{Wu}^{3}$, Jianfeng $\mathrm{Liu}^{4}$, \\ Seiki Sasaki ${ }^{5}$, Takahisa Yamada ${ }^{6}, \mathrm{Su} \mathrm{Ya}^{7}$, Huanmin $\mathrm{Zhou}^{3}$, Linsen Zan² ${ }^{2 *}$ Bin Tong ${ }^{1 \bullet}$
}

\footnotetext{
${ }^{1}$ The State Key Laboratory of Reproductive Regulation and Breeding of Grassland Livestock, School of Life Sciences, Inner Mongolia University, 010070 Hohhot, China

${ }^{2}$ College of Animal Science and Technology, National Beef Cattle Improvement Center,

Northwest A \& F University, 712100 Yangling, China

${ }^{3}$ Inner Mongolia Key Laboratory of Biomanufacture, College of Life Sciences, Inner Mongolia Agriculture University, 010010 Hohhot, China

${ }^{4}$ Key Laboratory of Animal Genetics, Breeding, and Reproduction, Ministry of Agriculture, National Engineering Laboratory for Animal Breeding, College of Animal Science and Technology, China Agricultural University, 100193 Beijing, China

${ }^{5}$ Maebashi Institute of Animal Science, Livestock Improvement Association of Japan, 371-0121 Maebashi, Japan

${ }^{6}$ Department of Agrobiology, Faculty of Agriculture, Niigata University, 950-2181 Niigata, Japan ${ }^{7}$ Inner Mongolia Animal Husbandry Station, 010010 Hohhot, China •Corresponding authors: zanlinsen@163.com; tongbin@imu.edu.cn

${ }^{\sharp}$ These authors contributed equally to this study
}

\begin{abstract}
Growth and carcass traits are the main breeding objectives in beef cattle. The aim of this study was to confirm genetic effects of the $c$. ${ }^{*} 188 G>A$ SNP of $A K I R I N 2$, the g.231054C $>T$ SNP of TTN, the $g .1471620 G>T$ SNP of $E D G 1$ and the g.70014208A $>G$ SNP of $M Y B P C 1$ on growth and carcass traits in Chinese Qinchuan (QC) cattle, as well as to compare the frequencies of the well-characterized alleles of these SNPs among six Chinese cattle populations, three Japanese cattle populations, two European cattle populations and one Korean cattle population. In this study, a total of 665 cattle samples were genotyped using MassARRAY and PCR-RFLP. Association analysis explored effects of four SNPs on growth and carcass traits including body length, wither height, hip height, hip width, rump length, chest depth, chest circumference, back fat thickness, ultrasound longissimus muscle area and ultrasound longissimus muscle depth in $\mathrm{QC}(\mathrm{P}<0.05$ to $\mathrm{P}<0.001)$. The well-characterized $A\left(c .{ }^{*} 188 G>A\right), T(g .231054 C>T)$ and $T($ g.1471620G $>T)$ alleles in Japanese Black cattle were significantly higher than Chinese cattle breeds; on the contrary, the $G$ allele
\end{abstract}

*This work was supported by grants from the Inner Mongolia Science \& Technology Plan (No. 20180260) of China, the "Grassland Meritocrat" Scholars Program (CYYC6068) of Inner Mongolia Autonomous Region, and the High-level Talents Import Program (No. 21400-5165112) of Inner Mongolia University of China, the High Level Overseas Talents Project ([2018]No.190) from Ministry of Human Resources and Social Security of China, also supported by grants from Key R\&D plan (2019NY-098) of Shaanxi Province and National Key R\&D Program (2018YFD0501700) of China. 
(g.70014208A $>G$ ) was markedly higher in Chinese cattle breeds than other cattle breeds. These results suggest that the four SNPs might be useful as a molecular marker for growth-related traits in Chinese QC cattle.

Key words: association, candidate genes, economic traits, SNPs, Qinchuan cattle

Growth and carcass traits are complex and controlled by multiple genes (Andersson, 2001; Andersson and Georges, 2004), which have an important effect on the economics of beef production. Thus, a better knowledge of the molecular architecture of growth and carcass traits is important as it may generate new opportunities for more effective marker-assisted selection, leading to economic benefits to the beef production industry (Raza et al., 2020 a).

To date, numerous candidate genes and molecular markers associated with economic traits of beef cattle have been identified, and some of them have already been used in cattle breeding (Raza et al., 2019; Yamada, 2014; Raza et al., 2020 b). Among them, the akirin 2 (AKIRIN2) gene is located within genomic regions of quantitative trait loci (QTLs) for marbling score and longissimus muscle area in Japanese Black (JB) cattle (Takasuga et al., 2007), as well as marbling score in Angus cattle (McClure et al., 2010); the titin (TTN) gene was found in the genomic regions within QTLs for marbling score, longissimus muscle area and subcutaneous fat thickness in JB (Takasuga et al., 2007), as well as subcutaneous fat thickness in a Brahman $\times$ Hereford sire developed half-sib family (Casas et al., 2003); the endothelial differentiation sphingolipid G-protein-coupled receptor 1 (EDG1) gene is located within genomic regions of QTLs for marbling score and body weight in JB (Takasuga et al., 2007), subcutaneous fat thickness in Angus (Mateescu et al., 2017), as well as marbling score in a Belgian Blue $\times$ MARC III developed half-sib family and a Piedmontese $\times$ Angus sire developed half-sib family (Casas et al., 2001), and the myosin binding protein $C$, slow type (MYBPC1) is included in the genomics regions of QTLs for rib thickness and subcutaneous fat thickness in JB (Takasuga et al., 2007), marbling score in Angus (McClure et al., 2010), hip height, rump length, rump width and chest depth in a cattle population including 1554 AI bulls distributed in 14 halfsib families (nine in Holstein, three in Normande and two in Montbéliarde breeds) (Boichard et al., 2003), as well as intramuscular fat in a Brangus heifers population (Peters et al., 2012). Thus, these genes could be considered as important candidate genes for carcass and growth traits in beef cattle. Furthermore, the $c$. ${ }^{*} 188 G>A$ single nucleotide polymorphism (SNP) in the 3' untranslated region (UTR) of AKIRIN2, the g.231054C $>T$ SNP in the promoter region of $T T N$, the $g .1471620 G>T$ SNP in the promoter region of $E D G 1$ and the $g .70014208 A>G$ SNP in the promoter region of $M Y B P C 1$, showed associations of these SNPs with growth or carcass traits in JB (Sasaki et al., 2009; Yamada et al., 2009 a, b, 2011; Tong et al., 2014 a, 2015) and Korean native (KN) (Kim et al., 2013) cattle. However, the relationships of these SNPs with growth and carcass traits in Chinese cattle breed have not been investigated.

Chinese indigenous cattle breeds can be divided into three groups based on their geographic distribution, morphological characteristics and sex chromosome poly- 
morphisms: the northern type in North China (NC), the central type in the middle (CC) and lower areas of the Yellow River and the southern type in South China (SC) (CNCAGR, 2011). The Qinchuan (QC) cattle, which is a typical breed of CC, are well-known to be good beef cattle in China, because of distinctive qualities including good adaptability and fine beef flavor among others. However, QC cattle exhibit a number of limitations compared to imported commercial beef cattle breeds, such as slow growth rate and underdeveloped hind hips. Accordingly, it is necessary to select important functional genes and molecular marker to increase the economic traits of Chinese native cattle (Tong et al., 2017; Wang et al., 2020).

Therefore, the objectives of this study reported herein were to: (1) perform association analyses of these SNPs in the AKIRIN2, TTN, EDG1 and MYBPC1 genes with growth and carcass traits in QC, (2) investigate the genetic diversity of these SNPs in three Chinese typical cattle groups (including QC, Mongolia cattle (MG), Luxi (LX), Wuling (WL) and Longlin (LL) cattle breeds), and (3) compare the frequencies of the well-characterized alleles of these SNPs in Chinese cattle breeds to those of JB, Japanese Brown (JBR), Japanese Short Horn (JSH), Holstein (HOL), Brown Swiss (BS) (Watanabe et al., 2010, 2011; Tong et al., 2014 b) and KN breeds (Kim et al., 2013).

\section{Material and methods}

\section{Ethics standards and animals}

The animals handling and experiments were conducted according to the guidelines laid down by Ministry of Science and Technology, 2004 China. The protocol was approved by the Institutional Animal Care and Ethics Committee of Inner Mongolia University, notified vide notification No.IMU-2018-01, dated 01-03-2018. The $41 \mathrm{MG}$ population from Inner Mongolia Autonomous Region of China (MGC) and 24 LX cattle were sampled from Chifeng Shengquan Ecological Animal Husbandry Co., Ltd. Ten milliliters of blood was collected from each cattle for DNA extraction.

\section{Samples and phenotypic data}

Genomic DNA of $41 \mathrm{MGC}$ and $24 \mathrm{LX}$ were extracted from blood samples with a TIANamp Blood DNA kit (TIANGEN Biotech, Beijing, China). The quality and quantity of the extracted DNA were evaluated using a Nanodrop ${ }^{\circledR}$ spectrophotometer (Thermo Fisher Scientific, Waltham, MA, USA) and by agarose gel electrophoresis. The DNA samples and phenotypes of 350 QC adult females (24-30 months, nonpregnant) were provided from Northwest A \& F University, Yangling, China (Wu et al., 2018; Raza et al., 2020 b, c, d). Animals were chosen at random from the National Beef Cattle Improvement Center herd (Yangling, China). All cows were fed the same total mixed ration with a roughage to concentrate ratio of $3: 2$. The herd was managed under the same conditions (temperature, humidity etc.). The feeding was offered based on NRC standards (Nutrient Requirement of Beef Cattle, 2016). The growth traits including body length, withers height, hip height, hip width, rump 
length, chest depth and chest circumference were estimated as per standard procedure (Wu et al., 2018; Raza et al., 2020 b, c, d). The carcass traits including back fat thickness, ultrasound loin muscle area and ultrasound loin muscle depth were estimated using ultrasound technology (Sono-grader ultrasound machine, Renco, USA). The phenotypic traits were measured through ultrasonography, because ultrasound measurement predicts carcass quality traits in live animals in a nondestructive manner. The ultrasonic probe was placed in the area between the 12th and 13th ribs (Wu et al., 2018; Raza et al., 2020 b, c, d). The DNA samples of 50 MG population from Mongolia (MGM), $50 \mathrm{WL}$ and $50 \mathrm{LL}$ cattle were provided from the Inner Mongolia Key Laboratory of Biomanufacture, Inner Mongolia Agriculture University. The information of cattle breeds in this study is shown in Table 1.

Table 1. Information of six cattle populations selected for genotyping

\begin{tabular}{lccc|c}
\hline \multicolumn{1}{c|}{ Breed } & Abbreviation & Number & Type \\
\hline $\begin{array}{l}\text { Mongolia cattle population (Inner Mongolia } \\
\text { Autonomous Region of China) }\end{array}$ & MGC & 41 & North China (NC) \\
Mongolia cattle population (Mongolia) & MGG & 50 & North China (NC) \\
Qinchuan cattle & QC & 350 & Central China (CC) \\
Luxi cattle & UM & 24 & Central China (CC) \\
Wuling cattle & WL & 50 & South China (SC) \\
Longlin cattle & LL & 50 & South China (SC) \\
\hline
\end{tabular}

\section{Genotyping using iPLEX MassARRAY}

For 350 QC cattle population, the $c$. ${ }^{*} 188 G>A$ SNP of $A K I R I N 2$, the $g .231054 C>T$ SNP of $T T N$, the $g .1471620 G>T$ SNP of $E D G 1$ and the $g .70014208 A>G$ SNP of $M Y B P C 1$ were genotyped with the MassARRAY ${ }^{\circledR}$ SNP genotyping system (Agena Bioscience, San Diego, CA, USA). PCR and extension primers were designed from sequences containing each target mutation and $\sim 100$ upstream and downstream bases with Assay Design Suite (http://agenabio.com/assay-design-suite-20-software) using the default settings. The genotype of each allele was analyzed using the Sequenom MassARRAY iPLEX platform (Gabriel et al., 2009). The resulting data was analyzed using the MassARRAY Typer 4.0 Analyzer software (Agena Bioscience, San Diego, CA, USA).

\section{Genotyping using PCR-RFLP}

For $41 \mathrm{MGC}, 50 \mathrm{MGM}, 50 \mathrm{WL}, 50 \mathrm{LL}$ and $24 \mathrm{LX}$ cattle populations, the c. ${ }^{*} 188 G>A$ SNP of $A K I R I N 2$, the $g .231054 C>T$ SNP of $T T N$, the $g .1471620 G>T$ SNP of EDG1 and the $g .70014208 A>G$ SNP of $M Y B P C 1$ were genotyped using PCR-restriction fragment length polymorphism (RFLP) method as described previously (Sasaki et al., 2009; Yamada et al., 2009 a, b; Tong et al., 2014 a). The PCR primers and restriction enzymes used for PCR-RFLP are shown in Table 2. 


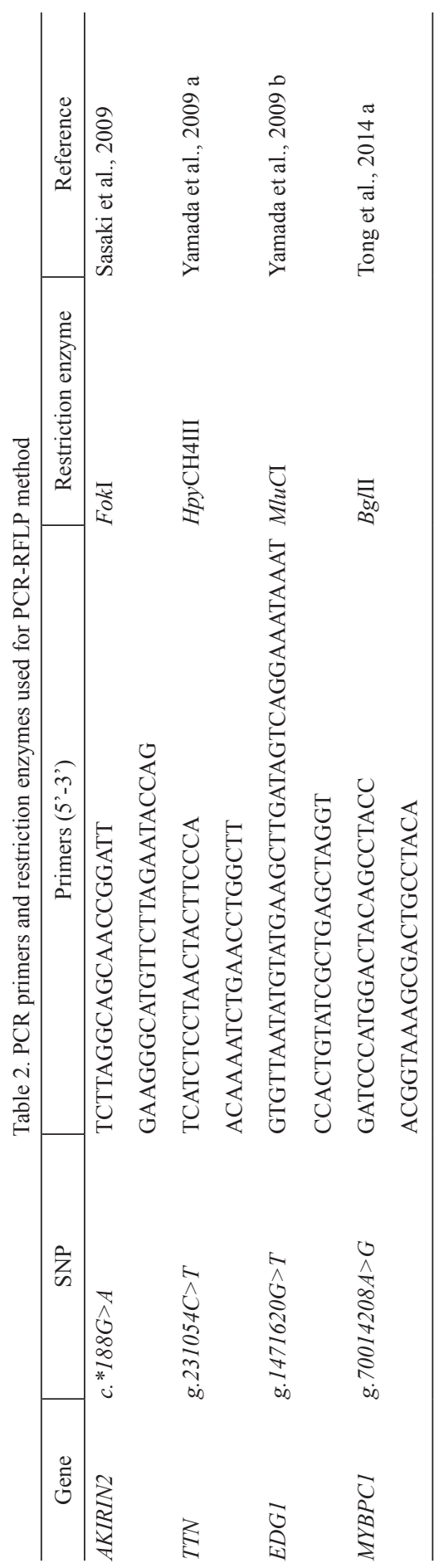




\section{Genetic diversity analysis}

Genotypic and allelic frequencies for all four SNPs of QC, MGC, MGG, WL, LL and LX cattle populations were calculated directly. Estimation of Hardy-Weinberg equilibrium was measured through $\chi^{2}$ test in PopGene software version 3.2. Population genetics indicators such as gene heterozygosity (He) and polymorphism information content (PIC) level were measured through established methods (Nei and Roychoudhury, 1974). The allelic frequencies of each SNP among cattle breeds or groups were compared by a $\chi^{2}$ test in Pop Gene software version 3.2 .

\section{Association analysis}

A General Linear Model (GLM) (SPSS 24.0, Chicago, USA) was used for the association analysis between SNPs and selected body and carcass traits in QC. The statistical linear model for this analysis was the same as previous reports (Wei et al., 2018; Raza et al., 2020 b, c, d):

$$
Y_{i j k}=\mu+G_{i}+A_{i}+A_{k}+e_{i j k}
$$

where:

$Y_{i j k}=$ trait value per individual,

$\mu=$ overall population mean per trait,

$G_{i}=$ fixed effect associated with genotype,

$A_{i}=$ fixed effect of age,

$A_{k}=$ fixed effect due to the age of dam,

$e_{i j k}=$ standard error.

The Bonferroni correction was used to adjust P values (Wei et al., 2018).

\section{Results}

\section{Genetic diversity of four SNPs in six cattle populations}

For the $c{ }^{*} 188 G>A$ SNP of AKIRIN2, the $g .231054 C>T$ SNP of $T T N$, the g. 1471620G>T SNP of EDG1 and the g.70014208A $>G$ SNP of $M Y B P C 1$, the frequencies of the two alleles and the three genotypes of each of four SNPs in the MGC, MGG, QC, LX, WL and LL cattle populations are listed in Table 3, as are the genetic indices (Ho, He, $n_{e}$ and PIC). No significant departures from the Hardy-Weinberg equilibrium at the $5 \%$ level were detected by any test for the four SNPs (Table 3 ). In this study, the LL population showed the lowest value of PIC in each of four SNPs compared to the other cattle populations. 


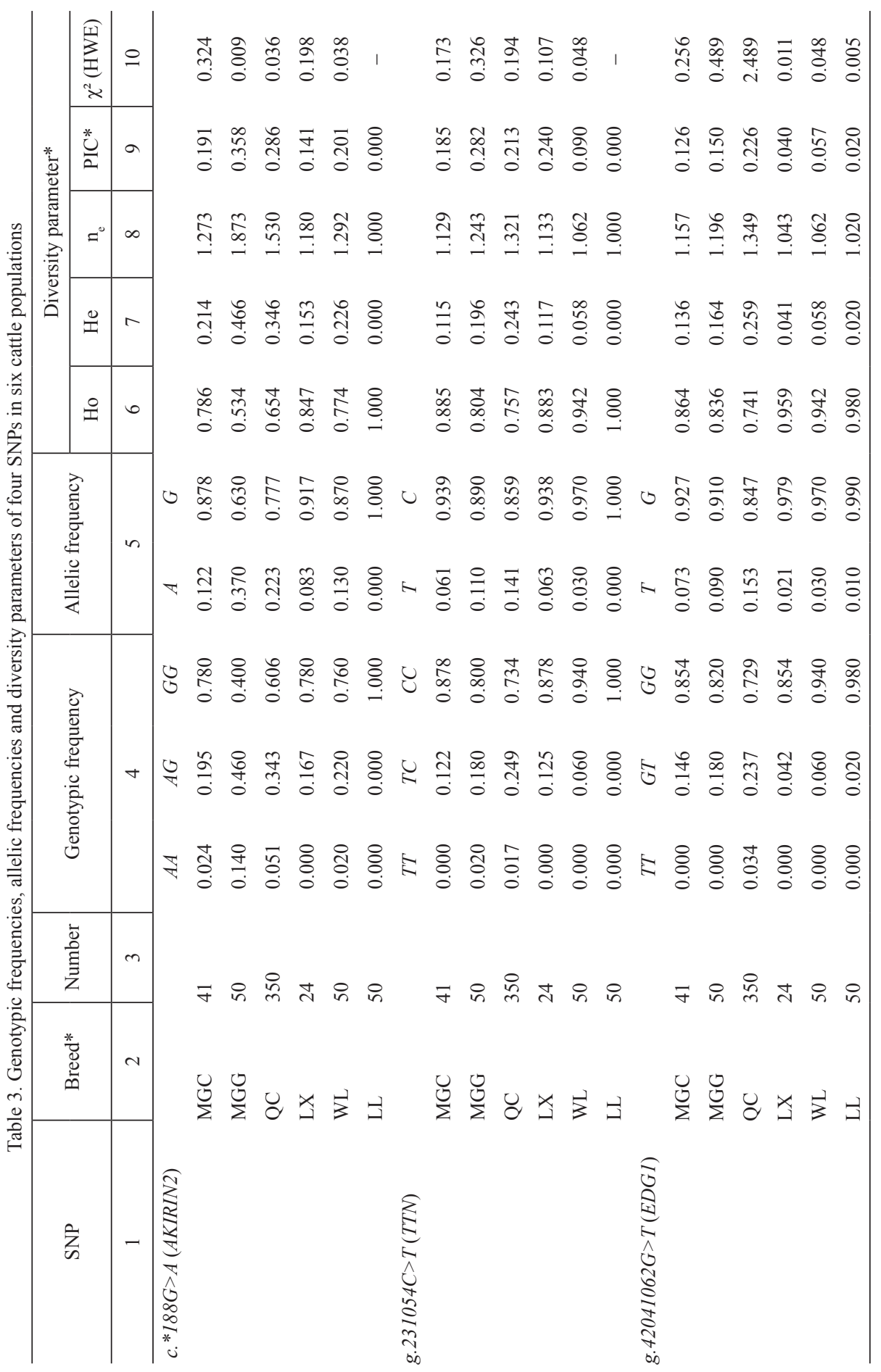




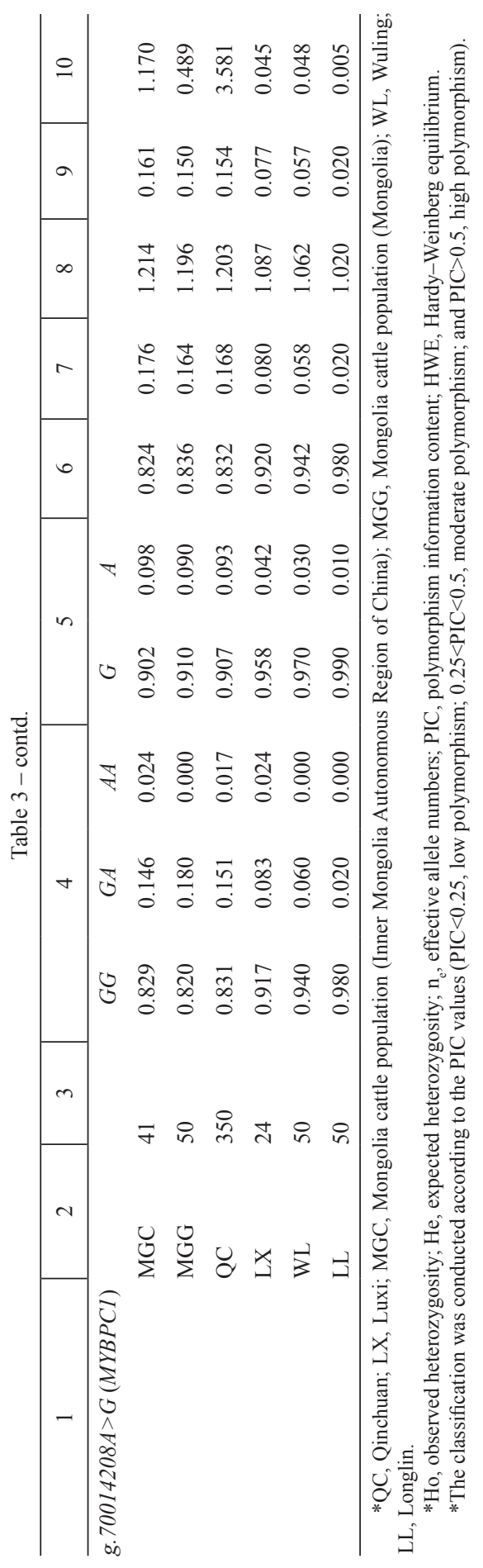




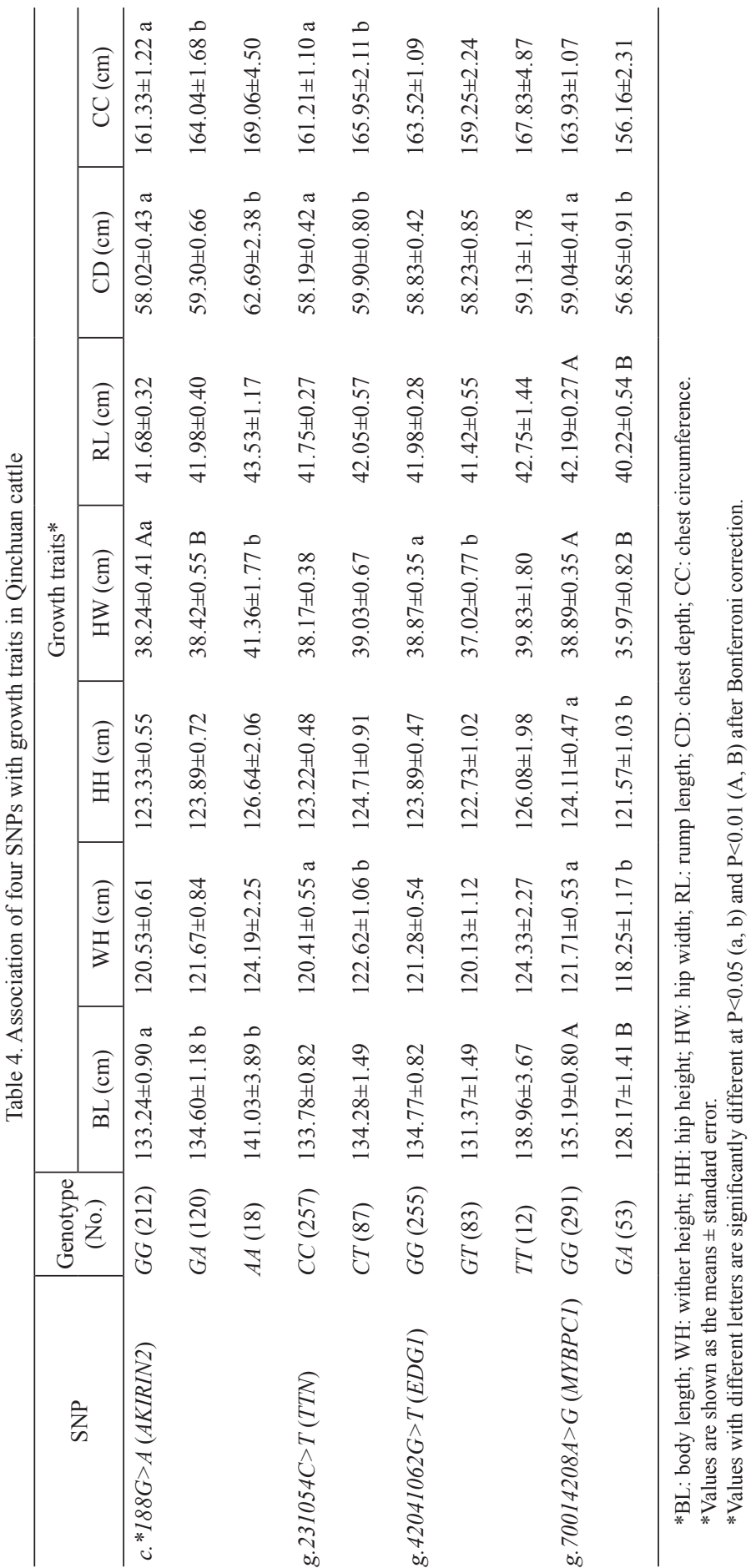




\section{Associations between four SNPs and growth traits in Qinchuan cattle}

The effects of the $c .{ }^{*} 188 G>A$ SNP of $A K I R I N 2$, the $g .231054 C>T$ SNP of $T T N$, the $g .1471620 G>T$ SNP of EDG1 and the $g .70014208 A>G$ SNP of MYBPC1 on growth traits were analyzed in 350 QC population (Table 4). For the $c$. ${ }^{*} 188 G>A$ SNP in $A K I R I N 2$, the individuals with $A A$ and $G A$ genotypes had significantly longer body length than the individuals with $G G$ genotype $(\mathrm{P}<0.05)$; the individuals with $A A$ and $G A$ had significantly greater hip width than the individuals with $G G(\mathrm{P}<0.05$, $\mathrm{P}<0.001$, respectively); and the genotypes of the $c . * 188 G>A$ SNP also had statistically significant effect on chest depth $(\mathrm{P}<0.05)$ and chest circumference $(\mathrm{P}<0.05)$ (Table 4). For the g.231054C $>T$ SNP in $T T N$, there were only six cattle with the $T T$ genotype, therefore, their associations and effects could not be reliably estimated and they were excluded from the analysis. The individuals with $C T$ genotype had significantly greater wither height $(\mathrm{P}<0.05)$, chest depth $(\mathrm{P}<0.05)$ and chest circumference $(\mathrm{P}<0.05)$ compared with the individuals with $C C$ genotype (Table 4$)$. For the g. 1471620G $>T$ SNP in $E D G 1$, there were significant differences between $G G$ and $G T$ genotype of hip width $(\mathrm{P}<0.05)$, whereas no association was detected in the other growth traits (Table 4). For the g.70014208A $>G$ SNP in $M Y B P C 1$, the only six cattle with $A A$ genotype were excluded from the analysis. Thus, the animals with $G G$ homozygotes exhibited significantly greater body length $(\mathrm{P}<0.001)$, wither height $(\mathrm{P}<0.05)$, hip height $(\mathrm{P}<0.05)$, hip width $(\mathrm{P}<0.01)$, rump length $(\mathrm{P}<0.01)$ and chest depth $(\mathrm{P}<0.05)$ compared to the animals with $G A$ heterozygotes (Table 4$)$.

Table 5. Association of four SNPs with carcass traits in Qinchuan cattle

\begin{tabular}{|c|c|c|c|c|}
\hline \multirow{2}{*}{ SNP } & \multirow{2}{*}{$\begin{array}{l}\text { Genotype } \\
\text { (No.) }\end{array}$} & \multicolumn{3}{|c|}{ Carcass traits* } \\
\hline & & BFT (cm) & $\operatorname{ULMA}\left(\mathrm{cm}^{2}\right)$ & ULMD $(\mathrm{cm})$ \\
\hline \multirow[t]{3}{*}{ c. ${ }^{*} 188 G>A(A K I R I N 2)$} & $G G(212)$ & $0.89 \pm 0.02$ & $47.12 \pm 1.14$ & $4.51 \pm 1.14$ \\
\hline & $G A(120)$ & $0.89 \pm 0.03$ & $47.44 \pm 1.34$ & $4.53 \pm 1.50$ \\
\hline & $A A(18)$ & $1.05 \pm 0.12$ & $47.57 \pm 3.33$ & $4.71 \pm 3.43$ \\
\hline \multirow[t]{2}{*}{ g.231054C >T (TTN) } & $C C(257)$ & $45.53 \pm 0.97 \mathrm{~A}$ & $0.88 \pm 0.02$ & $4.50 \pm 0.08$ \\
\hline & $C T(87)$ & $51.09 \pm 1.66 \mathrm{~B}$ & $0.94 \pm 0.03$ & $4.60 \pm 0.15$ \\
\hline \multirow[t]{3}{*}{ g. $42041062 G>T(E D G 1)$} & $G G(255)$ & $0.89 \pm 0.02 \mathrm{a}$ & $44.99 \pm 0.94 \mathrm{Aa}$ & $4.45 \pm 1.19$ \\
\hline & $G T(83)$ & $0.88 \pm 0.03 \mathrm{a}$ & $52.93 \pm 1.69 \mathrm{~B}$ & $4.77 \pm 1.68$ \\
\hline & $T T(12)$ & $1.13 \pm 0.16 \mathrm{~b}$ & $56.14 \pm 6.19 \mathrm{~b}$ & $4.53 \pm 1.29$ \\
\hline \multirow[t]{2}{*}{ g. 70014208A>G $(M Y B P C 1)$} & $G G(291)$ & $0.91 \pm 0.02$ & $47.37 \pm 0.95$ & $4.60 \pm 0.22 \mathrm{~A}$ \\
\hline & $G A(53)$ & $0.84 \pm 0.04$ & $47.83 \pm 1.97$ & $4.09 \pm 0.07 \mathrm{~B}$ \\
\hline
\end{tabular}

*BFT: back fat thickness; ULMA: ultrasound longissimus muscle area; ULMD: ultrasound longissimus muscle depth.

*Values are shown as the means \pm standard error.

*Values with different letters are significantly different at $\mathrm{P}<0.05$ (a, b) and $\mathrm{P}<0.01$ (A, B) after Bonferroni correction.

\section{Associations between four SNPs and carcass traits in Qinchuan cattle}

The results of association between the $c . * 188 G>A$ SNP of AKIRIN2, the $g .231054 C>T$ SNP of $T T N$, the $g .1471620 G>T$ SNP of $E D G 1$ and the g.70014208A>G SNP of $M Y B P C 1$ and carcass traits in 350 QC are shown in Ta- 
ble 5. For the $c .{ }^{*} 188 G>A$ SNP in AKIRIN2, no significant effect on the back fat thickness, ultrasound longissimus muscle area and ultrasound longissimus muscle depth was detected in QC (Table 5). For the g.231054C $>$ T SNP in TTN, the SNP genotype had the statistically significant effect on the back fat thickness $(\mathrm{P}<0.01)$, but not for ultrasound longissimus muscle area and ultrasound longissimus muscle depth in QC (Table 5). For the g.70014208A $>$ G SNP in MYBPC1, the ultrasound longissimus muscle depth of the $G A$ heterozygotes was significantly greater than that of the $G G$ homozygotes $(\mathrm{P}<0.001)$, but not for back fat thickness and ultrasound longissimus muscle area in QC cattle (Table 5). In addition, at the $g .1471620 G>T$ locus of $E D G 1$, the back fat thickness of the $T T$ homozygotes was significantly thinner than those of the $G T$ heterozygotes and $G G$ homozygotes $(\mathrm{P}<0.05)$. The ultrasound longissimus muscle area of the TT homozygotes was significantly larger than that of the $G G$ homozygotes $(\mathrm{P}<0.05)$, as well as that of the $G T$ heterozygotes was significantly larger than that of the $G G$ homozygotes $(\mathrm{P}<0.001)$ (Table 5).

Table 6. Statistical significance for differences in the allele frequencies of the four SNPs among the six groups of cattle populations

\begin{tabular}{|c|c|c|c|c|c|c|}
\hline \multirow[b]{2}{*}{ SNP } & \multirow[b]{2}{*}{ Group } & \multicolumn{5}{|c|}{ Group } \\
\hline & & $\begin{array}{l}\text { Central } \\
\text { China }\end{array}$ & $\begin{array}{l}\text { South } \\
\text { China }\end{array}$ & $\begin{array}{c}\text { Japanese } \\
\text { cattle }^{* 1}\end{array}$ & $\begin{array}{c}\text { European } \\
\text { cattle }^{* 1}\end{array}$ & $\begin{array}{c}\text { Korean native } \\
\text { cattle }^{* 2}\end{array}$ \\
\hline \multirow{5}{*}{ c. $* 188 G>A$ SNP $(A K I R I N 2)$} & North China & n.s. & $* * *$ & $* *$ & n.s. & $* * *$ \\
\hline & Central China & & $* * *$ & $* * *$ & n.s. & $* * *$ \\
\hline & South China & & & $* * *$ & $* * *$ & $* * *$ \\
\hline & Japanese cattle & & & & $* * *$ & n.s. \\
\hline & European cattle & & & & & $* * *$ \\
\hline \multirow[t]{4}{*}{ g.231054C $>T \mathrm{SNP}(T T N)$} & North China & n.s. & $* *$ & $* * *$ & $* * *$ & - \\
\hline & Central China & & $* * *$ & $* * *$ & $* * *$ & - \\
\hline & South China & & & $* * *$ & $* *$ & - \\
\hline & Japanese cattle & & & & $* * *$ & - \\
\hline \multirow[t]{4}{*}{ g.1471620G $>T \mathrm{SNP}(E D G 1)$} & North China & $*$ & $* *$ & $* * *$ & $* * *$ & - \\
\hline & Central China & & $* * *$ & $* * *$ & $* * *$ & - \\
\hline & South China & & & $* * *$ & $*$ & - \\
\hline & Japanese cattle & & & & $* * *$ & - \\
\hline \multirow{4}{*}{$\begin{array}{l}\text { g. } 70014208 A>G \text { SNP } \\
(M Y B P C 1)\end{array}$} & North China & n.s. & $* *$ & $* * *$ & $* * *$ & - \\
\hline & Central China & & $* * *$ & $* * *$ & $* * *$ & - \\
\hline & South China & & & $* * *$ & $* * *$ & - \\
\hline & Japanese cattle & & & & $* * *$ & - \\
\hline
\end{tabular}

*n.s.: non-significant; * $\mathrm{P}<0.05 ; * * \mathrm{P}<0.01 ; * * * \mathrm{P}<0.001$.

${ }^{*}$ The data of the $g .1471620 G>T$ SNP $(E D G 1), g .70014208 A>G$ SNP $(M Y B P C 1), c . * 188 G>A$ SNP $(A K I-$ RIN2) and g.231054C $>$ T SNP $(T T N)$ in Japanese and European cattle breeds were referred from Watanabe et al., 2010, 2011; Tong et al., 2014 b.

${ }^{* 2}$ The data of the $c .{ }^{*} 188 G>A$ SNP (AKIRIN2) in Korean native cattle was referred from Kim et al., 2013.

\section{Statistical comparisons of allele frequencies among six cattle groups}

To further analyze the distribution of the well-characterized allele of each of the four SNPs in the experimental cattle populations based on the different genetic background, the MGC, MGG, QC, LX, WL and LL of this study as well as the JB, 
JBR, JSH, HOL, BS and KN were divided into six groups which included North China (NC, including MGC and MGG), Central China (CC, including QC and LX), South China (SC, including WL and LL) (CNCAGR, 2011), Japanese cattle group (JG, including JB, JBR and JSH), European cattle group (EG, including HOL and $\mathrm{BS}$ ), and KN (Table 6). The distribution of the well-characterized allele frequency of each SNP in the cattle populations based on the different genetic background are shown in Figure 1. At the $c .{ }^{*} 188 G>A$ locus in AKIRIN2, the well-characterized $A$ allele frequency in the $\mathrm{JG}$ and $\mathrm{KN}$ was obviously higher than the other cattle groups (Table 6). At the $g .231054 C>T$ locus in $T T N$, the well-characterized $T$ allele frequency in the JG was significantly higher than the other cattle groups $(\mathrm{P}<0.001$, Table 6). At the $g .1471620 G>T$ locus in $E D G 1$, the well-characterized $T$ allele frequency in the JG was significantly higher than the other cattle groups $(\mathrm{P}<0.001$, Table 6). On the contrary, the distribution of the well-characterized $G$ allele frequency of the g.70014208A $>G$ SNP in MYBPC1 was significantly higher in NC, CC and $\mathrm{SC}$ than the JG and EG $(\mathrm{P}<0.001$, Table 6$)$. In addition, the well-characterized $T$ allele of the $g .231054 C>T$ SNP was almost absent in the SC and EG, the same to the well-characterized $T$ allele of the $g .1471620 G>T$ SNP in the SC and EG, as well as the well-characterized $G$ allele frequency of the $g .70014208 A>G$ SNP in the EG. The $\chi^{2}$ tests for different distribution of allele frequencies of the $c . * 188 G>A$ SNP in AKIRIN2, the g.231054C $>T$ SNP in TTN, the $g .1471620 G>T$ SNP in EDG1 and the g. 70014208A $>G$ SNP in MYBPC1 genes between any two cattle groups are listed in Tables S1-4.

(a)

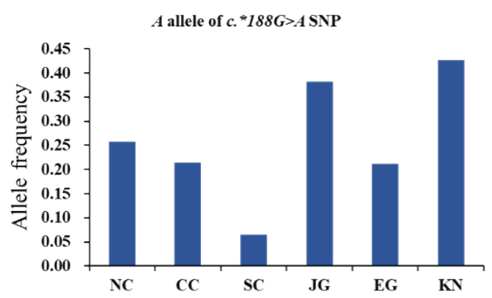

(c)

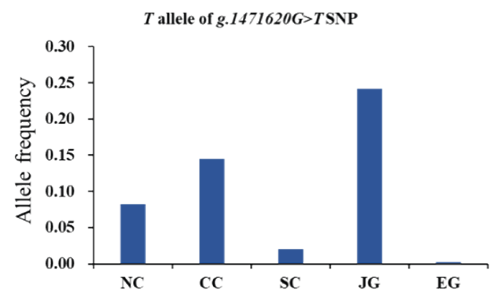

(b)

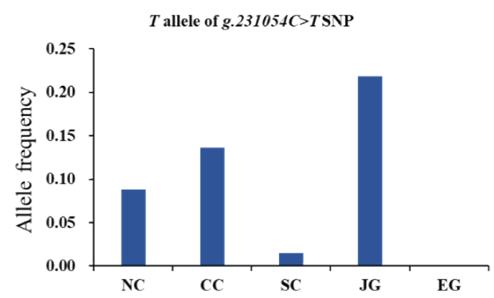

(d)

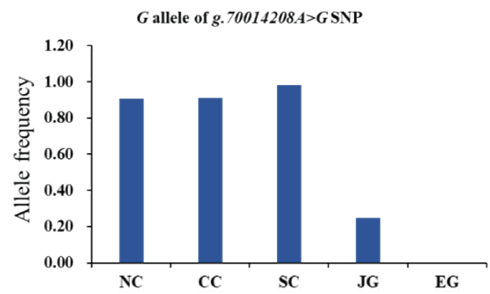

Figure 1. Distribution of the well-characterized allele of each SNP in the cattle populations based on the different genetic background. (a) The $A$ allele of $c$. ${ }^{*} 188 G>A$ SNP (b) The $T$ allele of $g .231054 C>T$

SNP (c) The $T$ allele of $g .1471620 G>T$ SNP (d) The $G$ allele of $g .70014208 A>G$ SNP. NC: North China; CC: Central China; SC: South China; JG: Japanese cattle group; EG: European cattle group; 


\section{Discussion}

The AKIRIN2, TTN, EDG1 and MYBPC1 genes showed different expression levels in the longissimus muscle between JB and HOL (Sasaki et al., 2006 a). These genes are located within genomic regions of QTLs for carcass and growth traits in JB, Angus and some crossbred populations (Takasuga et al., 2007; McClure et al., 2010; Casas et al., 2003; Mateescu et al., 2017; Casas et al., 2001; Boichard et al., 2003; Peters et al., 2012). The $c .{ }^{*} 188 G>A$ SNP in the 3 'UTR of AKIRIN2 showed associations with marbling score in JB (Sasaki et al., 2009) as well as marbling score and longissimus muscle area in KN (Kim et al., 2013). The proposed function of Akirins is as a transcription factor required for NF-jB-dependent gene expression in Drosophila and mice, and in the regulation of the innate immune response in Drosophila (Goto et al., 2008; Galindo et al., 2009). We speculate that the $c{ }^{*} 188 G>A$ SNP might affect mRNA stability to decrease the expression level of AKIRIN2, resulting in high growth performance in QC. The $g .231054 C>T$ SNP in the promoter region of TTN showed associations with marbling score and longissimus muscle area in JB (Yamada et al., 2009 a, 2011). Since TTN is known to be involved in myofibrillogenesis (Itoh-Satoh et al., 2002), we can hypothesize that the g. 231054C $>T$ SNP in the promoter region might have an impact on TTN expression and also growth performance by affecting TTN promoter activity in QC. The g. 1471620G $>T$ SNP in the promoter region of EDG1 showed association with marbling score in JB (Yamada et al., 2009 b), simultaneously one other SNP in EDG1 had effects on marbling score, subcutaneous fat thickness, carcass weight, longissimus muscle area and rib thickness (Yamada et al., 2009 c; Sukegawa et al., 2010). The EDG1 is known to be involved in blood vessel formation (Liu et al., 2000), The $g .1471620 G>T$ SNP might have an impact on EDG1 expression and also back fat thickness, ultrasound longissimus muscle area by affecting $E D G 1$ promoter activity in QC. The g.70014208A>G SNP in the promoter region of MYBPC1 had effects on marbling score and longissimus muscle area (Tong et al., 2014 a, 2015). The MYBPC1 interacts with muscle-type creatine kinase, potentially allowing it to regulate energy homeostasis during muscle contraction by coupling to the myofibril (Chen et al., 2011). Thus, the increase of the MYBPC1 expression level might lead to high growth performance through enhancing muscle satellite cell proliferation. We suggest that the $g .70014208 A>G$ SNP might have an impact on the MYBPC1 expression, and also growth performance by affecting the $M Y B P C 1$ promoter activity in QC.

Around the second century A.D., cattle migrated from North Asian continent via the Korean peninsula to Japan (Mukai et al., 1989). In Japanese cattle, both genetic (Namikawa, 1980) and morphological (Ogawa et al., 1989) studies have illustrated that native Japanese cattle are Bos taurus and are representatives of the "TuranoMongolian" type (Felius, 1995). The Korean native cattle (Hanwoo), is a cattle breed that is native to the Korean peninsula and the Japanese islands, which is considered to belong to the Bos taurus (Mannen et al., 1998, 2004; Seung et al., 2004). Actually, the JB has been subjected to strong selection for high marbling over the last 50 years, but not in other breeds such as JBR and JSH, as well as all Korean indigenous cattle 
breeds are just used for beef meat production (Sasaki et al., 2006 b) and consumer demands are driving efforts to increase meat production and produce higher quality meat in Korea (Han et al., 2011). Unlike them, the growth performance is regarded as the major breeding goal in most breeds of Chinese indigenous cattle. In this study, the frequencies of marbling-related $A$ allele (in the $c .{ }^{*} 188 G>A$ SNP of $\left.A K I R I N 2\right)$ in the JB and KN, $T$ allele (in the $g .231054 C>T$ SNP of $T T N$ ) in JB and $T$ allele (in the g. 1471620G $>T$ SNP of EDG1) in JB were significantly higher than in Chinese cattle breeds (Tables S1-S4). The probable reason could be the breeding for high marbling in JB and $\mathrm{KN}$ cattle breeds. Interestingly and conversely, the well-characterized $G$ allele (in the $g .70014208 A>G$ SNP of $M Y B P C 1$ ) was markedly higher in Chinese cattle breeds than JB (Table S4). Moreover, the $G$ allele (in the $g .70014208 A>G$ SNP of $M Y B P C 1$ ) was almost null in JBR and JSH; on the contrary, the $G$ allele was almost full in WL and LL breeds of CC (Table 3), likely due to genetic background. In addition, the PIC of each SNPs in HOL and BS of EG was related low or null, maybe due to the breeding aim for dairy-related traits in HOL and BS. We noted that well-known cattle breeds such as Angus and Shorthorn should be tested to explore the distribution of the well-characterized alleles in European beef cattle in the future study.

Recently, China cattle beef industry is growing rapidly to meet the meat demand of large population. Although a large number of molecular markers has been identified for breeding purpose, still more research is needed to explore more useful molecular markers for the cattle breed improvement programs (Tong et al., 2017). In the present study, association analysis showed effects of four SNPs on growth and carcass traits including body length, wither height, hip height, hip width, rump length, chest depth, chest circumference, back fat thickness, ultrasound longissimus muscle area and ultrasound longissimus muscle depth in Chinese QC cattle. However, future research is needed to confirm their effects in larger cattle populations.

\section{Conclusion}

The results of this study suggest that the $c{ }^{*} 188 G>A$ SNP of AKIRIN2, the $g .231054 C>T$ SNP of $T T N$, the $g .1471620 G>T$ SNP of EDG1 and the g. 70014208A $>G$ SNP of $M Y B P C 1$ genes might be useful as a specific DNA marker for growth and carcass traits in Chinese QC cattle.

\section{Conflict of interest}

The authors declare that they have no conflict of interest.

\section{Acknowledgements}

The authors thank Dr. Li Zhang (Inner Mongolia University) for their assistance with sample collection. The authors also thank the Chifeng Shengquan Ecological Animal Husbandry Co., Ltd. for providing purebred cattle breed samples. We gratefully acknowledge the assistance from Beijing Compass Biotechnology Co., Ltd. for MassARRAY analysis. 
Table S1. Statistical significance for differences in the A allele frequency of c. ${ }^{*} 188 \mathrm{G}>$ A SNP among 12 cattle populations

\begin{tabular}{|c|c|c|c|c|c|c|c|c|c|c|c|}
\hline Breed* & LX & MGC & MGG & WL & LL & $\mathrm{JB} * 1$ & $\mathrm{JBR}^{* 1}$ & $\mathrm{JSH}^{* 1}$ & HOL $* 1$ & $\mathrm{BS} * 1$ & $\mathrm{KN}^{* 2}$ \\
\hline QC & $*$ & * & $* *$ & * & $* * *$ & $* * *$ & $* * *$ & $* * *$ & n.s. & n.s. & $* * *$ \\
\hline LX & & n.s. & $* * *$ & n.s. & $* *$ & $* * *$ & $* * *$ & n.s. & * & $*$ & $* * *$ \\
\hline MGC & & & $* * *$ & n.s. & $* * *$ & $* * *$ & $* * *$ & $* *$ & n.s. & n.s. & $* * *$ \\
\hline MGG & & & & $* * *$ & $* * *$ & $*$ & $* *$ & $* * *$ & $* *$ & $* *$ & n.s. \\
\hline WL & & & & & $* * *$ & $* * *$ & $* * *$ & $* * *$ & n.s. & n.s. & $* * *$ \\
\hline LL & & & & & & $* * *$ & $* * *$ & n.s. & $* * *$ & $* * *$ & $* * *$ \\
\hline JB & & & & & & & n.s. & $* * *$ & $* * *$ & $* * *$ & n.s. \\
\hline JBR & & & & & & & & $* * *$ & $* * *$ & $* * *$ & $* * *$ \\
\hline JSH & & & & & & & & & $* * *$ & $* * *$ & $* * *$ \\
\hline HOL & & & & & & & & & & n.s. & $* * *$ \\
\hline BS & & & & & & & & & & & $* * *$ \\
\hline
\end{tabular}

*QC: Qinchuan; LX: Luxi; MGC: Mongolia cattle population (Inner Mongolia Autonomous Region of China); MGG: Mongolia cattle population (Mongolia); WL: Wuling; LL: Longlin; JB: Japanese Black; JBR: Japanese Brown; JSH: Japanese Short Horn; HOL: Holstein; BS: Brown Swiss; KN: Korean native cattle.

*n.s.: non-significant; $* \mathrm{P}<0.05 ; * * \mathrm{P}<0.01 ; * * * \mathrm{P}<0.001$.

${ }^{*}$ The data of the $c . * 188 G>A$ SNP (AKIRIN2) in Japanese and European cattle breeds were referred from Watanabe et al., 2011.

${ }^{* 2}$ The data of the $c . * 188 G>A$ SNP (AKIRIN2) in Korean native cattle was referred from Kim et al., 2013.

Table S2. Statistical significance for differences in the $T$ allele frequency of $g .231054 \mathrm{C}>\mathrm{T}$ SNP among 11 cattle populations

\begin{tabular}{|c|c|c|c|c|c|c|c|c|c|c|}
\hline Breed & LX & MGC & MGG & WL & LL & $\mathrm{JB}^{* 1}$ & $\mathrm{JBR}^{* 1}$ & $\mathrm{JSH}^{* 1}$ & HOL $* 1$ & $\mathrm{BS} * 1$ \\
\hline QC & n.s. & $*$ & n.s. & $* *$ & $* * *$ & $* * *$ & $* * *$ & n.s. & $* * *$ & $* * *$ \\
\hline LX & & n.s. & n.s. & n.s. & $*$ & $* *$ & $* *$ & n.s. & $* * *$ & $* * *$ \\
\hline MGC & & & n.s. & n.s. & $*$ & $* * *$ & $* * *$ & n.s. & $* * *$ & $* * *$ \\
\hline MGG & & & & $*$ & $* * *$ & $* *$ & $* *$ & n.s. & $* * *$ & $* * *$ \\
\hline WL & & & & & n.s. & $* * *$ & $* * *$ & n.s. & $* *$ & $* *$ \\
\hline LL & & & & & & $* * *$ & $* * *$ & $* *$ & - & - \\
\hline JB & & & & & & & n.s. & $* * *$ & $* * *$ & $* * *$ \\
\hline JBR & & & & & & & & $* * *$ & $* * *$ & $* * *$ \\
\hline JSH & & & & & & & & & $* * *$ & $* * *$ \\
\hline HOL & & & & & & & & & & - \\
\hline
\end{tabular}

*QC: Qinchuan; LX: Luxi; MGC: Mongolia cattle population (Inner Mongolia Autonomous Region of China); MGG: Mongolia cattle population (Mongolia); WL: Wuling; LL: Longlin; JB: Japanese Black; JBR: Japanese Brown; JSH: Japanese Short Horn; HOL: Holstein; BS: Brown Swiss.

*n.s.: non-significant; * $\mathrm{P}<0.05 ; * * \mathrm{P}<0.01 ; * * * \mathrm{P}<0.001$.

${ }^{*}$ The data of the g.231054C $>T$ SNP $(T T N)$ in Japanese and European cattle breeds were referred from Watanabe et al., 2011. 
Table S3. Statistical significance for differences in the $T$ allele frequency of g.1471620G $>$ T SNP among 11 cattle populations

\begin{tabular}{|c|c|c|c|c|c|c|c|c|c|c|}
\hline Breed* & LX & MGC & MMG & WL & LL & $\mathrm{JB}^{* 1}$ & $\mathrm{JBR}^{* 1}$ & $\mathrm{JSH}^{* 1}$ & $\mathrm{HOL}^{* 1}$ & BS*1 \\
\hline QC & * & n.s. & n.s. & $* * *$ & $* * *$ & $* * *$ & $* * *$ & $* * *$ & $* * *$ & $* * *$ \\
\hline LX & & n.s. & n.s. & n.s. & n.s. & $* * *$ & n.s. & n.s. & * & n.s. \\
\hline MGC & & & n.s. & n.s. & * & $* * *$ & n.s. & n.s. & $* * *$ & $* * *$ \\
\hline MGG & & & & n.s. & $* *$ & $* * *$ & n.s. & * & $* * *$ & $* * *$ \\
\hline WL & & & & & n.s. & $* * *$ & n.s. & n.s. & $* *$ & n.s. \\
\hline LL & & & & & & $* * *$ & n.s. & n.s. & n.s. & n.s. \\
\hline JB & & & & & & & $* * *$ & $* * *$ & $* * *$ & $* * *$ \\
\hline JBR & & & & & & & & n.s. & $* *$ & $*$ \\
\hline JSH & & & & & & & & & * & n.s. \\
\hline HOL & & & & & & & & & & n.s. \\
\hline
\end{tabular}

*QC: Qinchuan; LX: Luxi; MGC: Mongolia cattle population (Inner Mongolia Autonomous Region of China); MGG: Mongolia cattle population (Mongolia); WL: Wuling; LL: Longlin; JB: Japanese Black; JBR: Japanese Brown; JSH: Japanese Short Horn; HOL: Holstein; BS: Brown Swiss.

*n.s.: non-significant; * $\mathrm{P}<0.05 ; * * \mathrm{P}<0.01 ; * * * \mathrm{P}<0.001$.

${ }^{*}$ The data of the $g .1471620 G>T$ SNP $(E D G 1)$ in Japanese and European cattle breeds were referred from Watanabe et al., 2010.

Table S4. Statistical significance for differences in the $G$ allele frequency of $g .70014208 A>G$ SNP among 11 cattle populations

\begin{tabular}{|c|c|c|c|c|c|c|c|c|c|c|}
\hline Breed* & LX & MGC & MGG & WL & LL & $\mathrm{JB}^{* 1}$ & $\mathrm{JBR}^{* 1}$ & $\mathrm{JSH}^{* 1}$ & $\mathrm{HOL}^{* 1}$ & $\mathrm{BS} * 1$ \\
\hline QC & n.s. & n.s. & n.s. & * & $* *$ & $* * *$ & $* * *$ & $* * *$ & $* * *$ & $* * *$ \\
\hline LX & & n.s. & n.s. & n.s. & n.s. & $* * *$ & $* * *$ & $* * *$ & $* * *$ & $* * *$ \\
\hline MGC & & & n.s. & n.s. & $* *$ & $* * *$ & $* * *$ & $* * *$ & $* * *$ & $* * *$ \\
\hline MGG & & & & n.s. & $* *$ & $* * *$ & $* * *$ & $* * *$ & $* * *$ & $* * *$ \\
\hline WL & & & & & n.s. & $* * *$ & $* * *$ & $* * *$ & $* * *$ & $* * *$ \\
\hline LL & & & & & & $* * *$ & $* * *$ & $* * *$ & $* * *$ & $* * *$ \\
\hline JB & & & & & & & $* * *$ & $* * *$ & $* * *$ & $* * *$ \\
\hline JBR & & & & & & & & n.s. & n.s. & - \\
\hline JSH & & & & & & & & & n.s. & n.s. \\
\hline HOL & & & & & & & & & & n.s. \\
\hline
\end{tabular}

*QC: Qinchuan; LX: Luxi; MGC: Mongolia cattle population (Inner Mongolia Autonomous Region of China); MGG: Mongolia cattle population (Mongolia); WL: Wuling; LL: Longlin; JB: Japanese Black; JBR: Japanese Brown; JSH: Japanese Short Horn; HOL: Holstein; BS: Brown Swiss.

*n.s.: non-significant; * $\mathrm{P}<0.05 ; * * \mathrm{P}<0.01$; *** $\mathrm{P}<0.001$.

${ }^{* 1}$ The data of the $g .70014208 A>G$ SNP $(M Y B P C 1)$ in Japanese and European cattle breeds were referred from Tong et al., $2014 \mathrm{~b}$. 


\section{References}

A nde r s s o n L. (2001). Genetic dissection of phenotypic diversity in farm animals. Nat. Rev. Genet., 2: $130-138$.

Anderss on L., Georges M. (2004). Domestic-animal genomics: Deciphering the genetics of complex traits. Nat. Rev. Genet., 5: 202-212.

B o i ch ard D., Groh s C., B o urge o is F., C e rque ira F., F a ugeras R., N e a u A., R upp R., A m i g u e s Y., B o s cher M.Y., L e vé zi e l H. (2003). Detection of genes influencing economic traits in three French dairy cattle breeds. Genet. Sel. Evol., 35: 77-101.

C a sas E., S ton e R.T., Ke ele J.W., Shackelford S.D., Kappes S.M., Kooh maraie M. (2001). A comprehensive search for quantitative trait loci affecting growth and carcass composition of cattle segregating alternative forms of the myostatin gene. J. Anim. Sci., 79: 854-860.

Cas a E., Shackelford S.D., Ke ele J.W., Koohmaraie M., S m ith T.P.L., S to ne R.T. (2003). Detection of quantitative trait loci for growth and carcass composition in cattle. J. Anim. Sci., 81: 2976-2983.

Chen Z., Zh a o T.J., Li J., G a o Y.S., M eng F.G., Yan Y.B., Z hou H.M. (2011). Slow skeletal muscle myosin-binding protein-C (MYBPC1) mediates recruitment of muscle-type creatine kinase (CK) to myosin. Biochem. J., 436: 437-445.

China National Commission of Animal Genetic Resources (CNCAGR). (2001). Animal Genetic Resources in China: bovine. Beijing, China, China Agriculture Press, 15-20 pp.

Fe li u s M. (1995). Cattle breeds: An encyclopedia.

Gabrie 1 S., Z i a u g r a L., Ta b b a D D. (2009). SNP genotyping using the sequenom MassARRAY iPLEX platform. Curr. Protoc. Hum. Genet., John Wiley \& Sons, Inc.

Galindo R.C., Doncel-Perez E., Zivkovic Z., Naranjo V., Gortazar C., Mangold A.J., Martin-Hernand o M.P., Ko c an K.M., d e la Fuente J. (2009). Tick subolesin is an ortholog of the akirins described in insects and vertebrates. Dev. Comp. Immunol., 33: $612-617$.

Goto A., Matsushita K., Gesellchen V., E1 Chamy L., Kuttenkeuler D., Takeuchi O., Hoffmann J.A., Akira S., Boutros M., Reichhart J.M. (2008). Akirins are highly conserved nuclear proteins required for NF-kappa B-dependent gene expression in drosophila and mice. Nature Immunol., 9: 97-104.

H a n S.H., Cho I.C., Ko M.S., Kim E.Y., Oh H.S. (2011). A promoter polymorphism of MSTN g.-371T $>$ A and its associations with carcass traits in Korean cattle. Mol. Biol. Rep., 39: 3767-3772.

It oh-Sat oh M., Hayashi T., Nishi H., Koga Y., Arimura T., Koyanagi T., Takahashi M., Hohda S., Ueda K., Nouchi T., Hiroe M., Marumo F., Imaizumi T., Yasunami M., Kimura A. (2002). Titin mutations as the molecular basis for dilated cardiomyopathy. Biochem. Biophys. Res. Commun., 291: 385-393.

K i m H., L e e S.K., Hong M.W., P ark S.R., L e e Y.S., Ki m J.W., L e e H.K., J e ong D.K., S o n g Y.H., L e e S.J. (2013). Association of a single nucleotide polymorphism in the akirin 2 gene with economically important traits in Korean native cattle. Anim. Genet., 44: 750-753.

Li u Y., Wada R., Yamashita T., Mi Y., Deng C.X., Hobson J.P., Rosenfeldt H.M., Nava V.E., Chae S.S., Le e M.J., Liu C.H., Hla T., Spiegel S., Proia R.L. (2000). Edg-1, the G protein-coupled receptor for sphingosine-1-phosphate, is essential for vascular maturation. J. Clin. Invest. 106: 951-961.

Mannen H., Tsuji S., L oftus R.T., B radley D.G. (1998). Mitochondrial DNA variation and evolution of Japanese black cattle (Bos taurus). Genetics, 150: 1169-1175.

Mannen H., Kohno. M., N a g a t a Y., Ts u j i S., A m a no T. (2004). Independent mitochondrial origin and historical genetic differentiation of north eastern Asian cattle. Mol. Phylogenet. Evol., 32: 539-544.

M a t e e s c u R.G., G a r r i ck D.J., R e e c y J.M. (2017). Network analysis reveals putative genes affecting meat quality in Angus cattle. Front. Genet., 8: 171.

M c Clure M.C., M or s c i N.S., S chn ab el R.D., K i m J.W., Y a o P., Rolf M.M., M c K a y S.D., Gregg S.J., Ch a p p le R.H., N or th c u t t S.L., T a y lor J.F. (2010). A genome scan for quantitative trait loci influencing carcass, post-natal growth and reproductive traits in commercial Angus cattle. Anim. Genet., 41: 597-607. 
Mukai F., Tsuji S., Fukazaw a K., Ohtaga ki S., N a mbu Y. (1989). History and population structure of a closed strain of Japanese Black cattle. J. Anim. Breed. Genet., 106: 254-264.

$\mathrm{N}$ a m i k a w a T. (1980). Genetic aspects of domestication and phylogeny in cattle. Jap. J. Zootech. Sci., 51: $235-246$.

National Academies of Sciences, Engineering, and Medicine (2016). Nutrient requirements of beef cattle, 8th rev. ed. Washington D.C, USA, The National Academies Press.

N e i M., R o y c houdhury A.K. (1974). Sampling variances of heterozygosity and genetic distance. Genetics, 76: 379-390.

O gawa Y., Daigo M., A mas aki H. (1989). Craniometrical estimation of the native Japanese Mishima cattle, using multivariate analysis. Anat. Anz., 168: 197-202.

Peters S.O., Kizilkaya K., Garrick D.J., Fernando R.L., Re e y J.M., We aber R.L., S ilve r G.A., Th o m a s M.G. (2012). Bayesian genome-wide association analysis of growth and yearling ultrasound measures of carcass traits in Brangus heifers. J. Anim. Sci., 90: 3398-3409.

R a z a S., Kh a n R.,A b d e 1 n o u r S.A.,A b d E.M., K h a fag a A.F., Ta ha A., O h r a n H., M e i C., S chreurs N.M., Z a n L. (2019). Advances of molecular markers and their application for body variables and carcass traits in Qinchuan cattle. Genes (Basel), 10: 717.

Raza S., Khan S., Amjadi M., Abdelnour S.A., Ohran H., A lanazi K.M., Abd E.M., Taha A.E., Khan R., Gong C., Schreurs N.M., Zhao C., Wei D., Zan L. (2020 a). Genome-wide association studies reveal novel loci associated with carcass and body measures in beef cattle. Arch. Biochem. Biophys., 694: 108543.

R a z a S., Li u G.Y.,Z h o u L., G u i L.S., K h a n R., J in m e n g Y., C h u g a n g M., S c h r e u r s N.M., Ji R., Z a n L. (2020 b). Detection of polymorphisms in the bovine leptin receptor gene affects fat deposition in two Chinese beef cattle breeds. Gene, 758: 144957.

Raza S., Khan R., S chreurs N.M., Gu o H., Gu i L.S., M e i C., Z a n L. (2020 c). Expression of the bovine KLF6 gene polymorphisms and their association with carcass and body measures in Qinchuan cattle (Bos taurus). Genomics, 112: 423-431.

Raza A., Shijun L., Khan R., Schreurs N.M., Manzari Z., Abd E1-Aziz A.H., U1$1 \mathrm{ah}$ I., Kaster N., Shah M.A., Z an L. $(2020$ d). Polymorphism of the PLIN1 gene and its association with body measures and ultrasound carcass traits in Qinchuan beef cattle. Genome, 63: $483-492$.

S a s a ki Y., Nagai K., N a g a t a Y., D oronbek ov K., Nishimura S., Yoshioka S., Fuji ta T., Shiga K., Miyake T., Taniguchi Y., Yamada T. (2006 a). Exploration of genes showing intramuscular fat deposition-associated expression changes in musculus longissimus muscle. Anim. Genet., 37: 40-46.

S a s a ki Y., Miyake T., Gaillard C., Oguni T., Oht a gaki S. (2006 b). Comparison of genetic gains per year for carcass traits among breeding programs in the Japanese Brown and the Japanese Black cattle. J. Anim. Sci., 84: 317-323.

Sasaki S., Yamada T., Sukegawa S., Miyake T., Fujita T., Morita M., Ohta T., Takahagi Y., Murakami H., Morimatsu F., S a s a ki Y. (2009). Association of a single nucleotide polymorphism in akirin 2 gene with marbling in Japanese Black beef cattle. BMC Res. Notes, 2: 131.

S e ung S.Y., J i Y.S., S e on O.W., Hyung W.J., Ke un C.K., D ong S.S. (2004). Genetic relationship of Korean cattle (Hanwoo) based on nucleotide variation of mitochondrial D-loop region. Korean J. Genet., 26: 297-307.

Sukegawa S., Miyake T., Takahagi Y., Murakami H., Morimatsu F., Yamada T., S a s a ki Y. (2010). Replicated association of the single nucleotide polymorphism in EDG1 with marbling in three general populations of Japanese Black beef cattle. BMC Res. Notes, 3: 66.

Takasuga A., Wat anabe T., Mizoguchi Y., Hirano T., Ihara N., Takano A., Yokouchi K., Fujikawa A., Chiba K., Kobayashi N., Tatsuda K., Oe T., Furukawa-Kuroiwa M., Nishimura-Abe A., Fujita T., Inoue K., Mizoshita K., Ogino A., S u g i m o t o Y. (2007). Identification of bovine QTL for growth and carcass traits in Japanese Black cattle by replication and identical-by-descent mapping. Mamm. Genome, 18: 125-136.

Tong B., S a saki S., Muramatsu Y., Ohta T., Kos e H., Yamashiro H., Fujita T., Ya$\mathrm{m}$ a d a T. (2014 a). Association of a single-nucleotide polymorphism in myosin-binding protein $\mathrm{C}$, slow-type (MYBPC1) gene with marbling in Japanese Black beef cattle. Anim. Genet., 45: 611-612. 
Tong B., S as aki S., Muramatsu Y., Ohta T., Kose H., Fujita T., Yamada T. (2014 b). The G allele at the g.70014208A $>\mathrm{G}$ in the MYBPC1 gene associated with high marbling in Japanese Black cattle is at a low frequency in breeds not selected for marbling. J. Genet., 93: 231-233.

Tong B., Xing Y.P., Muramatsu Y., Ohta T., Kose H., Zhou H.M., Yamada T. (2015). Association of expression levels in skeletal muscle and a SNP in the MYBPC1 gene with growthrelated trait in Japanese Black beef cattle. J. Genet., 94: 135-137.

To ng B., Z hang L., Li G.P. (2017). Progress in the molecular and genetic modification breeding of beef cattle in China. Hereditas, 39: 984-1015.

Wang L., R a za S., Gu i L., Li S., Li u X., Yang X., Wang S., Z a n L., Z ha o C. (2020). Associations between UASMS2 polymorphism in leptin gene and growth, carcass and meat quality traits of cattle: a meta-analysis. Anim. Biotechnol., 17: 1-10.

Wat a nabe N., Yamada T., Yoshioka S., It on M., S a toh Y., Furuta M., Kom atsu S., S u m i o Y., F u j it a T., S a saki Y. (2010). The T allele at the g.1471620G $>$ T in the EDG1 gene associated with high marbling in Japanese Black cattle is at a low frequency in breeds not selected for marbling. Anim. Sci. J., 81: 142-144.

Watanabe N., Satoh Y., Fujita T., Ohta T., Kose H., Muramatsu Y., Yamamoto T., Yamada T. (2011). Distribution of allele frequencies at TTN g.231054C >T, RPL27A g. 3109537C $>$ T and AKIRIN2 c. 188G $>$ a between Japanese Black and four other cattle breeds with differing historical selection for marbling. BMC Res. Notes, 4: 10 .

We i D.W., Raza S.H.A., Zhang J.P., Gu i L.S., Rahman S.U., Khan R., Hosse in i S., K a le ri H., Z a n L.S. (2018). Polymorphism in promoter of SIX4 gene shows association with its transcription and body measurement traits in Qinchuan cattle. Gene, 656: 9-16.

Wu S., Wang Y., N ing Y., Gu o H., Wang X., Zhang L., Khan R., Cheng G., Wang H., $\mathrm{Z}$ a n L. (2018). Genetic variants in STAT3 promoter regions and their application in molecular breeding for body size traits in Qinchuan cattle. Int. J. Mol. Sci., 19: 1035.

Y a m a d a T. (2014). Genetic dissection of marbling trait through integration of mapping and expression profiling. Anim. Sci. J., 85: 349-355.

Yamada T., Sasaki S., Sukegawa S., Yoshioka S., Takahagi Y., Morita M., Murakami H., Morimatsu F., Fujita T., Miyake T., S as aki Y. (2009 a). Association of a single nucleotide polymorphism in titin gene with marbling in Japanese Black beef cattle. BMC Res. Notes, 2: 78.

Yamada T., Sasaki S., Sukegawa S., Miyake T., Fujita T., Kose H., Morita M., Takahagi Y., Murakami H., Morimatsu F., Sasaki Y. (2009 b). Novel SNP in 5' flanking region of EDG1 associated with marbling in Japanese Black beef cattle. Anim. Sci. J., 80: 486-489.

Yamada T., Itoh M., Nishimura S., Taniguchi Y., Miyake T., Sasaki S., Yoshiok a S., F u j it a T., Shig a K., M orit a M., S a s a ki Y. (2009 c). Association of single nucleotide polymorphisms in the endothelial differentiation sphingolipid G-protein-coupled receptor 1 gene with marbling in Japanese Black beef cattle. Anim. Genet., 40: 209-216.

Yamada T., S as aki S., Sukegawa S., Yoshioka S., Takahagi Y., Morita M., Murakami H., Morimatsu F., Fujita T., Miyake T., S as aki Y. (2011). Possible association of single nucleotide polymorphism in titin gene with growth-related traits in Japanese Black beef cattle. J. Anim Vet. Adv., 10: 2603-2606.

Received: 21 X 2020

Accepted: 15 IV 2021 